\title{
Surface Granular flows: Two Related Examples
}

\author{
D. V. Khakhar and Ashish V. Orpe \\ Department of Chemical Engineering, Indian Institute of Technology - Bombay, Powai, Mumbai, 400076, India \\ J. M. Ottino \\ Department of Chemical and Mechanical Engineering, \\ Northwestern University, Evanston, IL 60208, USA
}

\begin{abstract}
Granular surface flows are common in industrial practice and natural systems, however, theoretical description of such flows is at present incomplete. Two prototype systems involving surface flow are compared: heap formation by pouring at a point and rotating cylinders. Continuum models for analysis of these flows are reviewed, and experimental results for quasi-2d systems are presented. Experimental results in both systems are well described by continuum models.
\end{abstract}

\section{INTRODUCTION}

Granular flows have been the subject of considerable recent work [1-5] driven by both technological needs [6, 7] and the recognition that many aspects of the basic physics are poorly understood [8]. Surface flows of granular materials, that is flows confined to a surface layer on a static granular bed, are important in industrial practice and nature. Industrial examples appear in the transportation, processing and storage of materials in systems such as rotary kilns, tumbling mixers, and feeding and discharge of silos. Examples in nature include the formation of sand dunes, lava flow, avalanches, and transport of sediments in rivers. Although considerable progress has been made, theoretical description of surface flows is incomplete at present. Several approaches, based on different assumptions about the physics of the flows, have been proposed [9-19]. A few experimental studies are also available [9,19-33]. Most work is focussed on two systems: heap flow and rotating cylinder flow shown schematically in Fig. 1.

An important feature of surface granular flows is the interchange of particles between the flowing layer and the fixed bed. In the case of a rotating cylinder the interchange rate is determined by kinematics since the velocity of the fixed bed at the bed-layer interface is known. The situation in the case of heap flow is more complicated. Bouchaud et al. 12 proposed a phenomenological model (BCRE model) in which the interchange rate is determined by the local surface angle. A variation of this model proposed by Boutreux et al. [16] (BRdG model) has been broadly validated by continuum models [18, 19] and experiments 19], as we show below. Continuum models developed previously, for both heaps and rotating cylinders, are all based on depth-averaged hydrodynamic equations and differ primarily in the constitutive equations used. All the models contain parameters which must be evaluated from experiments, but in most cases, these parameters have not been determined.

*Electronic address: khakhar@iitb.ac.in
Here we present here a common continuum based framework for the analysis of both heap flow and rotating cylinder flow. The treatment closely follows that given in refs. 19] and 30]. Model predictions are compared to experimental results and to predictions of previous models. The general continuum model is presented first. Results for the heap formation and rotating cylinder flow are given next followed by conclusions.

\section{GENERAL CONTINUUM MODEL}

Consider a flowing layer on the surface of a granular bed assuming the flow is nearly uni-directional in the layer and curvature effects are small. The depth averaged continuity equation and the $x$-momentum balance equation are simplified using the following assumptions. The bulk density in the layer $(\rho)$ is nearly constant (since the dilation of the flowing particles is not too large in the relatively slow flows being considered). The velocity profile in the layer is linear and of the form [31,32] $v_{x}=2 u(y / \delta)$, where $u(x, t)$ is the depth averaged velocity in the layer and $\delta$ is the layer thickness. Slow plastic deformation [33] is neglected. The shear stress at the interface is taken to be 30

$$
\left.\tau_{x y}\right|_{y=0}=\rho d^{2} f(\rho)\left(\frac{\partial v_{x}}{\partial y}\right)^{2}-\rho g \delta \cos \beta \tan \beta_{s}
$$

where $d$ is the particle diameter and $\tan \beta_{s}$ is the effective coefficient of dynamic friction, with $\beta_{s}$ taken to be the static angle of repose. The stress is sensitively dependent on the local bulk density and based on recent empirical evidence [30] we take $f(\rho)=c \delta / d$ with $c \approx 1.5$. The governing equations then reduce to

$$
\begin{aligned}
\frac{\partial \delta}{\partial t}+\frac{\partial}{\partial x}(\delta u) & =-\Gamma, \\
\frac{\partial}{\partial t}(\delta u)+\frac{4}{3} \frac{\partial}{\partial x}\left(\delta u^{2}\right) & =-4 c d \frac{u^{2}}{\delta}+g \delta \frac{\sin \left(\beta-\beta_{s}\right)}{\cos \beta_{s}},(3)
\end{aligned}
$$

where $\Gamma$ is the flux from the layer into the bed. Further, assuming the static friction forces at the heap-layer interface to be fully mobilized, the Mohr-Coulomb criterion 


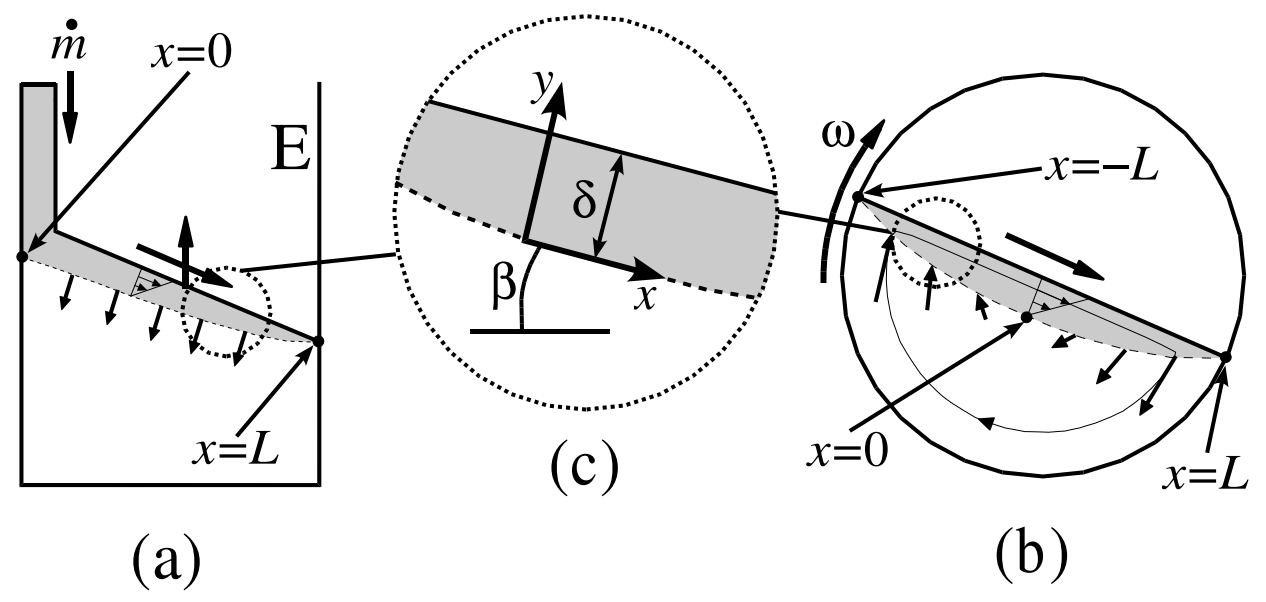

FIG. 1: Schematic view of surface flow systems: (a) Heap flow (b) Rotating cylinder flow. (c) Coordinate system used in the analysis.

yields

$$
\left.\tau_{x y}\right|_{y=0}=-\rho g \delta \cos \beta \tan \beta_{m},
$$

where $\tan \beta_{m}$ is the effective coefficient of static friction. Using eq. (11) and the assumptions given above, eq. (4) yields

$$
u=\dot{\gamma} \delta / 2
$$

with the shear rate given by

$$
\dot{\gamma}=\left[\frac{g \cos \beta \sin \left(\beta_{m}-\beta_{s}\right)}{c d \cos \beta_{m} \cos \beta_{s}}\right]^{1 / 2} .
$$

A similar analysis is given by Douady et al. 18] with the difference that no stress constitutive equation such as eq. (11) is used and instead the shear rate in the flowing layer is assumed to be constant.

\section{HEAP FORMATION}

Consider a quasi-steady flow $(\partial \delta / \partial t, \partial u / \partial t \approx 0)$ and a slowly varying interface angle $(\partial \beta / \partial x \approx 0)$ during heap formation. The continuity equation (eq.21) together with eq. (5) then reduces to

$$
\dot{\gamma} \delta \frac{\partial \delta}{\partial x}=-\Gamma,
$$

and the momentum balance equation (eq. 3) together with eq. (4) simplifies to

$$
\dot{\gamma}^{2} \delta \frac{\partial \delta}{\partial x}=-\frac{g \sin \left(\beta_{m}-\beta\right)}{\cos \beta_{m}} .
$$

Combining eqs. (7) and (8) yields $\Gamma=g \sin \left(\beta_{m}-\right.$ $\beta) / \dot{\gamma} \cos \beta_{m}$, which, for the case when $\beta_{m} \approx \beta$, reduces to

$$
\Gamma \approx V\left(\beta_{m}-\beta\right)
$$

where $V=g / \dot{\gamma} \cos \beta_{m}$. Thus, the continuum model yields a source term similar to the BRdG model; the scaling of $V$ is also similar to the BRdG model.

We further simplify the above equations for two different geometries of heap formation: closed, as shown in Fig. [1 and apen in which the end wall (E, Fig. Ta) is removed. In the open system at steady state, all the material entering the system leaves at the far edge of the heap and no particles are absorbed or eroded. This implies that $\Gamma=0$, which on substituting into eq. (9) $\beta=\beta_{m} \equiv$ constant. Using these results in eqs. (7) and (8) shows that the average velocity $(u)$ and thickness $(\delta)$ of the flowing layer are also constant in open systems. The mass flow rate in the system is given by $\dot{m}=\rho u \delta T$, where $T$ is the width of the layer. This expression, together with eq. (5), gives the following relationship between the layer thickness and mass flow rate

$$
\delta=[2 \dot{m} /(T \rho \dot{\gamma})]^{1 / 2}
$$

Experimental results [19] based on flow visualization studies validate the above predictions, and sample results are given below. Fig. 2 shows the variation of the maximum angle of repose with mass flow rate in the system for $2 \mathrm{~mm}$ steel balls in an open heap system (filled symbols). The data indicate that $\beta_{m}$, and thus the coefficient of static friction at the heap-layer interface $\left(\tan \beta_{m}\right)$, is not a constant but increases with the local flow rate. An increase in surface angle with flow rate was also reported by Lemieux and Durian 29]. Fig. 20 shows the variation of the layer thickness $(\delta)$ with mass flow rate. The solid line is a fitted curve of the form $\delta \propto \dot{m}^{1 / 2}$. This indicates agreement with theoretical predictions (eq. 10) if the product $\rho \dot{\gamma}$ is independent of mass flow rate.

In a closed system (Fig. 10), at steady state we must have $\Gamma \equiv$ constant for the heap to rise uniformly. Integrating eq. (7), the layer thickness profile is obtained as 

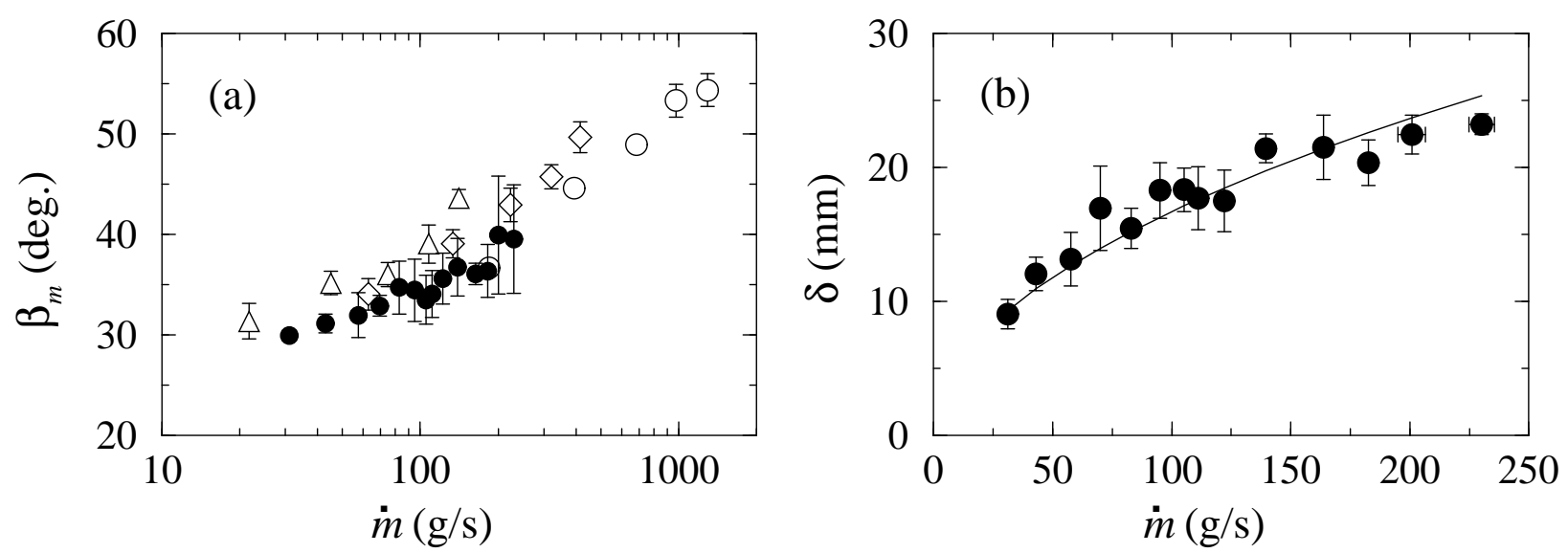

FIG. 2: Variation of the (a) maximum angle of repose $\left(\beta_{m}\right)$ and (b) layer thickness $(\delta)$ with mass flow rate $(\dot{m})$ for 2 mm steel balls. Filled symbols: open heap system [19]. Open symbols: rotating cylinder system for three cylinder sizes [30]. The solid line in (b) is a best fit of the form $\delta \propto \dot{m}^{1 / 2}$

$$
\delta=\left[\delta_{L}^{2}+2 \Gamma(L-x) / \dot{\gamma}\right]^{1 / 2}
$$

where $\delta_{L}$ is the layer thickness at the end of the layer, $x=L$, and $L$ is the length of the interface (Fig. T). The rise velocity is related to the mass flow rate by

$$
\Gamma=\dot{m} /(T L \rho),
$$

and the interface angle is calculated from eq. (9).

Experimental results [19] for closed systems show that the rise velocity $(\Gamma)$ varies nearly linearly with mass flow rate in agreement with eq. (12), and the bulk density, which is found to be constant, is $\rho=3.2 \mathrm{~g} / \mathrm{cm}^{3}$. Fig. 3 shows the variation of both interface angle $(\beta)$ and layer thickness $(\delta)$ with length along the bed-layer interface $(L-x)$ for a fixed mass flow rate. The solid line in Fig. 3b is a fit of eq. (111). There is a good match between the fitted line and the experimental data, which suggests that the shear rate, $\dot{\gamma}$, is constant. Similar results are obtained for all flow rates studied. Using experimental results for the rise velocity $(\Gamma)$ and the interface length (L), we obtain $\dot{\gamma}=20 \pm 2 \mathrm{~s}^{-1}$ from eq. (11), where the standard deviation indicated is calculated for all 10 flow rates studied. Using the value of the bulk density obtained above, we find from eq. (10) that the shear rate for the open system is $\dot{\gamma}=22 \pm 3 \mathrm{~s}^{-1}$. The value of the shear rate predicted from eq. (6) is $\dot{\gamma}=20 \pm 5 \mathrm{~s}^{-1}$ for the range of mass flow rates considered. Thus the shear rates for the open and closed systems are the same within experimental error, and predictions of theory are in reasonable agreement with experimental values.

\section{ROTATING CYLINDER}

The simplest case corresponds to rotating cylinder flow for $50 \%$ fill fraction. Assuming a nearly flat interface, the source term is given by $\Gamma=\omega x$. Substituting into the continuity equation (eq. (2) and integrating we obtain

$$
u \delta=\frac{\omega}{2}\left(L^{2}-x^{2}\right) .
$$

Using eq. (2) the momentum balance equation (eq. 3) simplifies to

$$
u \frac{d u}{d x}=\frac{3 g}{4} \frac{\sin \left(\beta-\beta_{s}\right)}{\cos \beta_{s}}-3 \frac{c d u^{2}}{\delta^{2}}+\frac{\omega x u}{\delta} .
$$

We consider two different limiting solutions to eqs. (13) and (14) below.

Firstly, consider the case when shear rate $(\dot{\gamma})$ is nearly constant. Using eq. (5), the flux equation (eq. 13) gives the layer thickness profile as

$$
\delta=\left(\frac{\omega}{\dot{\gamma}}\right)^{1 / 2}\left(L^{2}-x^{2}\right)^{1 / 2}
$$

which is symmetric for all rotational speeds $(\omega)$. Eq. (15) corresponds to the model of Makse [17], in which the shear rate is assumed to be a fitting parameter. In the present case the shear rate is obtained from eq. (6) and the mean velocity is given by $u=\dot{\gamma} \delta / 2$. Substituting these results in eq. (14), and using the Mohr-Coulomb condition (eq. (4) yields eq. (9) with $\Gamma=\omega x$ and $\beta_{m} \approx \beta$. This allows for calculation of the angle $(\beta)$ along the interface. Thus the assumption of a constant shear rate is consistent with the model, and gives a complete description of the flow. However, it is not apparent from the analysis, under what conditions the solution is valid.

Consider next the case when the shear rate is not constant along the layer, but when the acceleration $(d u / d x)$ is small. Eliminating $\delta$ using eq. (13), the scaled momentum balance becomes

$$
\bar{u} \frac{d \bar{u}}{d \xi}=\frac{3}{4 F r} \frac{\sin \left(\beta-\beta_{s}\right)}{\cos \beta_{s}}-12 c s \frac{\bar{u}^{4}}{\left(1-\xi^{2}\right)^{2}}+\frac{2 \xi \bar{u}^{2}}{1-\xi^{2}},
$$



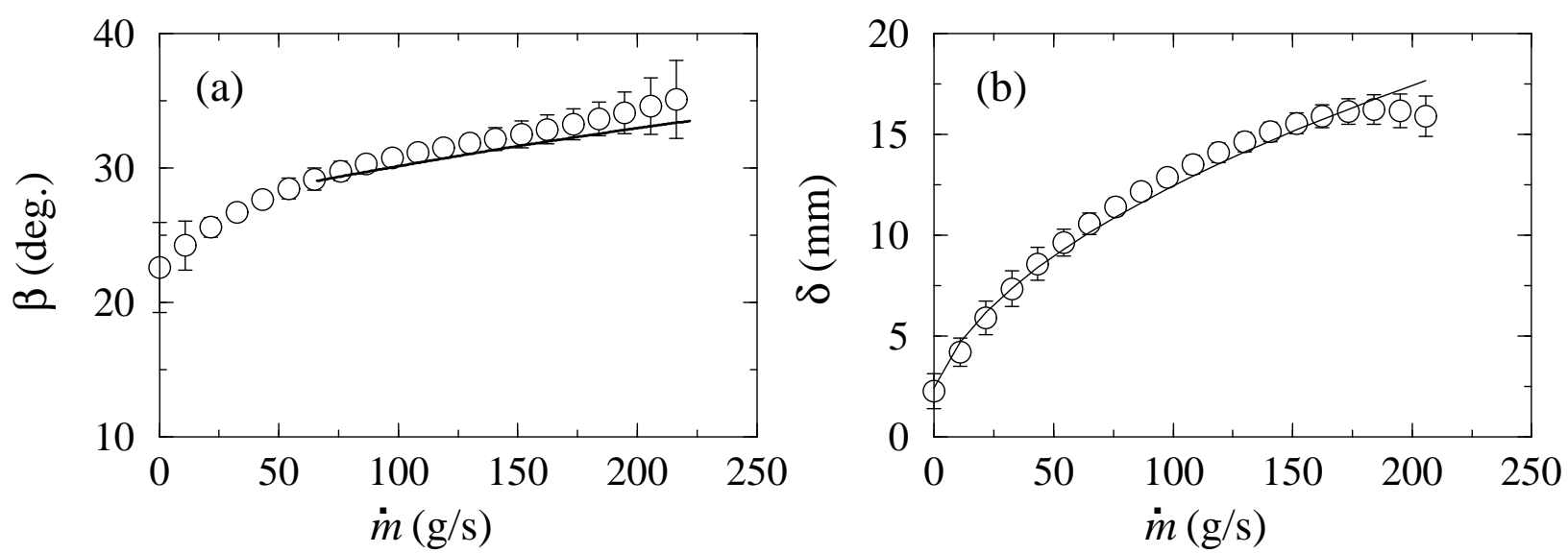

FIG. 3: Variation of the (a) surface angle $(\beta)$ and (b) layer thickness $(\beta)$ with distance from the edge of the heap $(L-x)$ for flow of $2 \mathrm{~mm}$ steel balls in a closed system. Symbols are experimental data and error bars indicate the standard deviation over six measurements. Solid line in (a) is a fit of eq. (9) and in (b) is the prediction of eq. (11).

where $\bar{u}=u / \omega L, \xi=x / L$ and the dimensionless parameters are the Froude number, $\mathrm{Fr}=\omega^{2} L / g$, and the size ratio, $s=d / L$. The first term on the right hand side of eq. (16) is the net driving force, that is the gravitational force less the frictional resistance to flow, and is independent of the flow velocity $(\bar{u})$. The second term is the 'viscous' resistance due to collisional stresses, and the third term arises as a result of in-flow and out-flow of particles from the layer. Both these terms depend on the flow velocity. Typical experimental Froude numbers for experiments in rotating cylinders are in the range $O\left(10^{-3}\right)$ to $O\left(10^{-2}\right)$. In these cases the driving force term $(O(1 / F r))$ is much larger than the acceleration term $(O(\xi / \sqrt{s F r})$ based on eq. 5$)$, particularly near the midpoint of the layer $(\xi=0)$. The collisional stress term is of the same magnitude as the net driving force term since the flow velocity increases to balance the two. Thus for $\xi \sqrt{\mathrm{Fr} / \mathrm{s}} \ll 1$ the acceleration term may be neglected.

For negligible acceleration $(d \bar{u} / d \xi \approx 0)$, the scaled mean flow velocity is obtained from eq. (16) as

$$
\bar{u}=\left(\frac{1-\xi^{2}}{12 c s}\right)^{1 / 2}\left[\xi+\left(\xi^{2}+9 c s A / F r\right)^{1 / 2}\right]^{1 / 2},
$$

where $A=\sin \left(\beta-\beta_{s}\right) / \cos \beta_{s}$. Using eq. (13), the scaled layer thickness profile is

$$
\bar{\delta}=\left[\frac{3 \operatorname{cs}\left(1-\xi^{2}\right)}{\xi+\left(\xi^{2}+9 \operatorname{cs} A / F r\right)^{1 / 2}}\right]^{1 / 2}
$$

where $\bar{\delta}=\delta / L$. The above solution is valid only if $A>0$, that is if $\beta>\beta_{s}$. For $\beta \leq \beta_{s}$, we have $\bar{u}=\bar{\delta}=0$, thus there is no steady flow possible if the interface angle is less the static angle of repose. This is consistent with the definition of the static angle of repose. Note that the layer profile is not symmetric about $\xi=0$, and for any $\xi>0$ we have $\bar{\delta}(-\xi)>\bar{\delta}(\xi)$, that is, the upper part of the layer $(\xi<0)$ is thicker than the lower part. The source of the asymmetry is the in-flow/out-flow term in the momentum balance (third term on the right hand side of eq. [16). In the upper part of the layer $(\xi<0)$ the flow is retarded by material entering the layer from the bed $(\Gamma<0)$ and the reverse is true in the lower part of the layer. Thus, the layer is thicker in upper part because of the lower velocity relative to the lower part of the layer $(\xi>0)$, resulting in a skewed profile. Further, eq. (18) indicates that the profile becomes more skewed with increasing Froude number $(F r)$ and decreasing size ratio $(s)$. In the limit, $F r / s \ll 1$, the scaled layer thickness profile becomes $\bar{\delta}=(\operatorname{csFr} / A)^{1 / 4}\left(1-\xi^{2}\right)^{1 / 2}$, which is identical to the result obtained assuming a constant shear (eq. 15) when eq. (6) is used to calculate the shear rate. This implies that a profile symmetric about the layer midpoint $(\xi=0)$ is obtained at very low Froude numbers and relatively high size ratios, and in this limit the shear rate is nearly constant.

The interface angle profile $(\beta(\xi))$ is obtained from eq. (9), using $\Gamma=\omega x$ and eq. (6) as

$$
\beta(\xi)=\beta_{m}-\frac{F r \cos \beta_{m}}{3 c s}\left[\xi+\left(\xi^{2}+9 c s A / F r\right)^{1 / 2}\right]^{1 / 2} \xi .
$$

In simplifying the preceding equation we assume $\beta_{m} \approx$ $\beta \approx \beta_{s}$. Eq. (19) indicates that the interface angle decreases monotonically with distance along the interface and at $\xi=0, \beta=\beta_{m}$. Thus in the rotating cylinder flow the maximum angle of repose can be experimentally obtained by measuring the interface angle at the midpoint of the layer. For $(\mathrm{Fr} / s) \xi$ sufficiently large and $\xi>0$, we get $\beta<0$, that is, for small size ratios and large Froude numbers the layer profile may turn up at the end. Conversely, when $(F r / s) \ll 1$, eq. (19) yields $\beta \approx \beta_{m}$, and the interface profile is nearly flat. Neglecting terms $O(\xi F r / s)$, which is consistent with the approximation in the momentum balance equation, we get $A=\left(\beta_{m}-\beta_{s}\right) / \cos \beta_{s}$. 

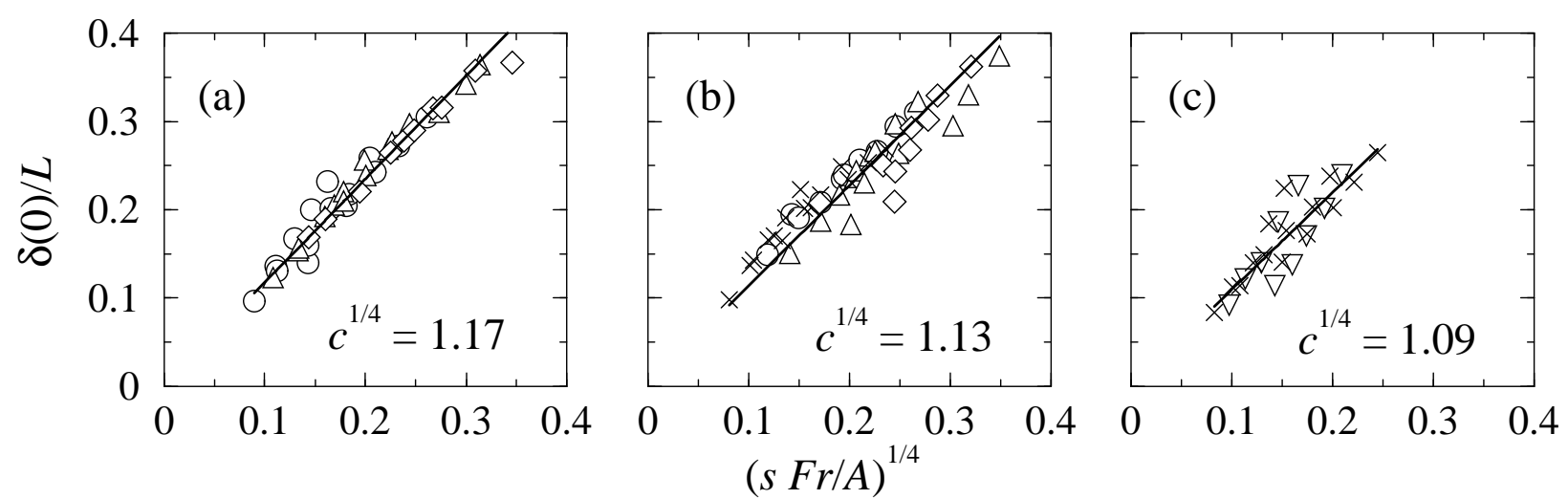

FIG. 4: Variation of the layer thickness at the midpoint $(\delta(0))$ with $(s F r / A)^{1 / 4}$ for (a) steel balls, (b) glass beads (c) and sand particles in cylinders of different sizes and at different rotational speeds. Symbols are experimental data for different sized particles: $\circ d=1 \mathrm{~mm}, \triangle d=2 \mathrm{~mm}, \diamond d=4 \mathrm{~mm}, \nabla d=0.4 \mathrm{~mm}$ and $\times d=0.8 \mathrm{~mm}$. The solid line is a fit of eq. (18) and the values of the parameter $c^{1 / 4}$ are indicated.
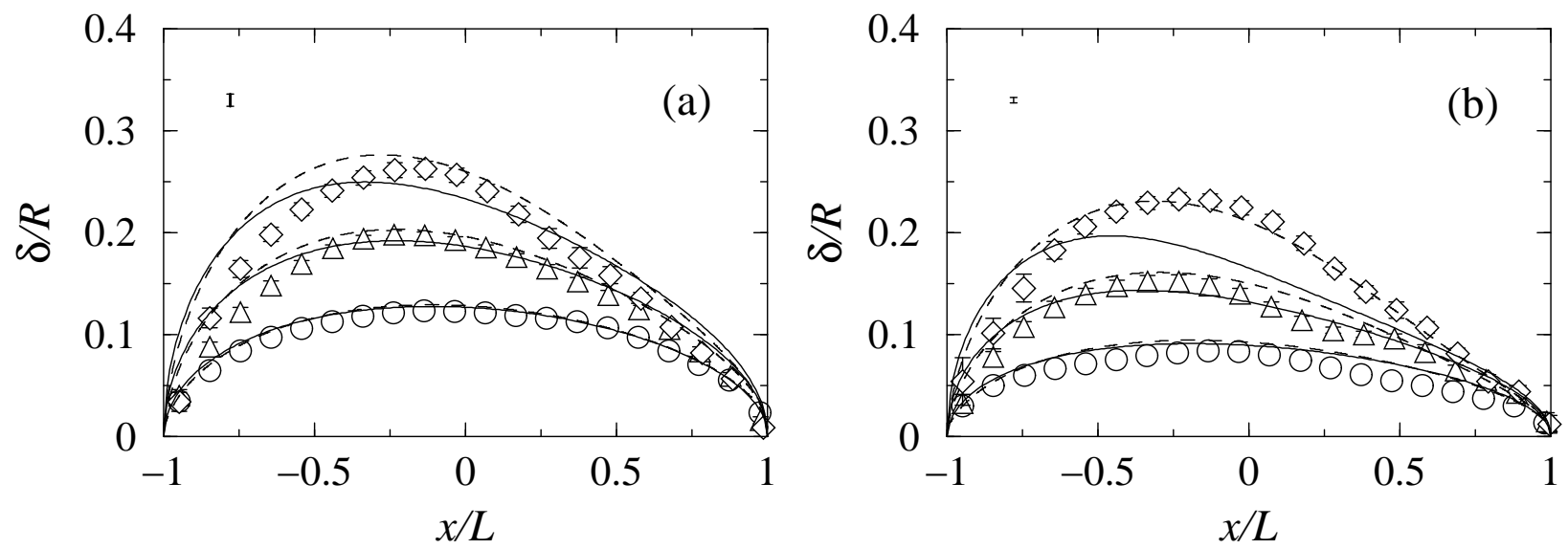

FIG. 5: Layer thickness profiles for (a) $2 \mathrm{~mm}$ steel balls and (b) $0.8 \mathrm{~mm}$ sand. Symbols denote experimental data for three different froude numbers $(F r): \circ F r=2 \times 10^{-3}, \triangle F r=22 \times 10^{-3}, \diamond F r=64 \times 10^{-3}$. Solid lines are predictions of eq. (18) and dashed lines are the predictions of the model of Khakhar et al. [13]. The error bars give the standard deviation over 6 measurements and the bar indicates the scaled diameter of a particle $(s=d / R)$.

Consider next a comparison of the theoretical results to experimental data. A few key numbers are reported, as they convey a sense of qualitative agreement. However, for full details the reader is referred to [30]. The model parameters required are $\beta_{s}, \beta_{m}$ and $c$. Data of Orpe and Khakhar 30 for the first two parameters are shown in Fig. 2a (open symbols) for $2 \mathrm{~mm}$ steel balls in rotating cylinders of 3 sizes and for different rotational speeds of the cylinders. The data correlates reasonably well with the mass flow rate at the midpoint of the layer calculated from $\dot{m}=\rho \omega L^{2} T / 2$, where $T$ is the cylinder length and the same density as in the heap experiments $(\rho=3.2$ $\mathrm{g} / \mathrm{cm}^{3}$ ) is used. Data spanning nearly two decades of flow rate fall on a single curve, although with some scatter. The maximum angle of repose increases with mass flow rate, and the measured values are similar to those from heap experiments which are also shown in the same figure. The static angle of repose is the angle at $\dot{m}=0$.

Orpe and Khakhar [30 had obtained $c \approx 1.5$ by fitting the theory of Khakhar et al. 13. to experimental layer thickness profiles. We obtain a new estimate of the parameter based on the layer thickness at the midpoint of the layer $(\xi=0)$, which, from eq. (18), is $\bar{\delta}(0)=(\operatorname{csFr} / A)^{1 / 4}$. Fig. 团 shows experimental data for $\bar{\delta}(0)$ versus $(s F r / A)^{1 / 4}$ for experimental data for 90 experiments comprising steel balls, glass beads and sand of different sizes in cylinders of different sizes and for different rotational speeds. The data falls on a straight line for each material (although with some scatter) and a least squares fit gives $c=1.9$ for steel balls, $c=1.6$ for glass beads and $c=1.4$ for sand. Since the model is essentially exact at $\xi=0$, the good fit implies that the proposed constitutive equation for stress is reasonable, and the shear rate in the layer is well-described by 
eq. (6) at $\xi=0$.

Predictions of the model for the layer thickness profile are compared to experimental data in Fig. 15 for sand particles and steel balls for different Froude numbers in a cylinder of radius $16 \mathrm{~cm}$, using the value of $c$ obtained above and experimental values for $\beta_{m}$ and $\beta_{s}$. The agreement is good except at the highest $F r$ and low $s$, and the all the qualitative features of the data are reproduced. At low $F r$ and relatively high $s$ studied, the profile is nearly symmetric (steel balls at the lowest $F r$ ), and the profiles become more skewed with increasing $F r$ and decreasing $s$. The deviation at the high Froude numbers and low size ratio are due to neglect of the acceleration term. Similar agreement is obtained for the other cases studied as well. The predictions of the model of Khakhar et al. 13] are shown in the figure as dashed lines. These nearly coincide with the results from the present model, except for the highest $F r$ for sand, indicating that the approximations made are reasonable for the parameter values of interest. It is remarkable that such a simple theory is able to describe the behavior of the system over such a wide range of parameters: materials include steel balls, glass beads and sand; varying shapes with steel balls being spherical, glass beads, nearly spherical and sand being irregularly shaped; size ratios in the range $s \in(0.005,0.05)$ and Froude numbers in the range $F r \in\left(2 \times 10^{-3}, 64 \times 10^{-3}\right)$. Model predictions of the interface angle profile are in reasonable agreement with experiments [30].

\section{CONCLUSIONS}

A theoretical framework serves to unify the behaviour of surface flows for two prototypical systems: heap flow and rotating cylinder flow. The model is based on a stress constitutive equation and failure criterion which contain three material parameters: $\beta_{s}, \beta_{m}$ and $c$. Analytical results for both systems give a complete description of the systems in terms of the layer thickness profiles $(\delta(x))$, average velocity of flow $(u(x))$ and the interface angle profile $(\beta(x))$. In open heap systems a layer of uni- form thickness with a uniform flow velocity is obtained, whereas in the closed heap system $\delta^{2} \propto x$. The interface angle is constant and equal to the maximum angle of repose in the open system, whereas it decreases with distance from the pouring point in the closed system. Results for the rotating cylinder are obtained for the case when the acceleration of particles in the layer is small $(\xi \sqrt{F r / s} \ll 1)$. The layer profile is found to be asymmetric about the midpoint of the layer $(\xi=0)$ with the upper part of the layer $(\xi<0)$ being thicker. The skewness increases with increasing Froude numbers and decreasing size ratios. The scaled shear rate $(\dot{\gamma} / \omega)$ decreases with increasing Froude number and size ratio. The layer interface angle decreases with distance in the flow direction. For high $\xi F r / s$ and $\xi>0$ the layer turns up, whereas when $\xi F r / s$ is small a nearly flat interface is obtained.

Quasi-2d experiments carried out for open and closed heaps and rotating cylinders of different sizes, by and large, validate the predictions of the theory. The three material parameters of the model $\left(\beta_{s}, \beta_{m}\right.$ and $\left.c\right)$ are all obtained from relatively simple measurements. The model equation can thus be applied to more complex geometries. Deviations of the model from experimental data appear in the interface angle profile in the rotating cylinder flow. This is most likely due to end wall effects which are discussed in 30].

\section{Acknowledgements}

D. V. Khakhar acknowledges the financial support of the Department of Science and Technology, India, through the Swarnajayanti Fellowship project (DST/SF/8/98) for part of this work. This work was supported in part by grants to J. M. Ottino from the Division of Basic Energy Sciences of the Department of Energy, the National Science Foundation, Division of Fluid and Particulate Systems, and the Donors of the Petroleum Research Fund, administered by the American Chemical Society.
[1] C. S. Campbell, Annu. Rev. Fluid Mech. 22, 57, (1990).

[2] H. M. Jaeger, S. R. Nagel, and R. P. Behringer, Rev. Mod. Phys. 68, 1259, (1996).

[3] J. Duran, Powder and Grains, Springer-Verlag, NewYork, (2000).

[4] G. H. Ristow, Pattern Formation in Granular Materials, Springer, Berlin, (2000).

[5] J. M. Ottino and D. V. Khakhar, Annu. Rev. Fluid Mech. 32, 55, (2000).

[6] B. J. Ennis, J. Green, and R. Davis, Chem. Eng. Prog. 90, 32, (1994)

[7] J. Bridgewater, Chem. Eng. Sci. 50, 4081, (1995).

[8] P. G. de Gennes, Rev. Mod. Phys. 71, S374, (1999).

[9] J. Rajchenbach, Phys. Rev. Lett. 65, 2221, (1990).
[10] S. J. Rao, S. K. Bhatia, and D. V. Khakhar, Powder Technol. 67, 153, (1991).

[11] O. Zik, D. Levine, S. G. Lipson, S. Shtrikman, and J. Stavans, Phys. Rev. Lett. 73, 644, (1994).

[12] J. P. Bouchaud, M. Cates, J. Ravi Prakash and S. Edwards, J. Phys. France I, 4, 1383, (1994).

[13] D. V. Khakhar, J. J. McCarthy, T. Shinbrot, and J. M. Ottino, Phys. Fluids, 9, 31, (1997).

[14] A. A. Boateng and P. V. Barr, J. Fluid Mech. 330, 233, (1997).

[15] T. Elperin and A. Vikhansky, Europhys. Lett. 42, 619, (1998).

[16] T. Boutreux, E. Raphaël, and P. G. de Gennes, Phys. Rev. E, 58, 4692, (1998). 
[17] H. A. Makse, Phys. Rev. Lett. 83, 3186, (1999).

[18] S. Douady, B. Andreotti and A. Daerr, Eur. Phys. J. 11, 131, (1999)

[19] D. V. Khakhar, A. V. Orpe, P. Andresén and J. M. Ottino, J. Fluid Mech. 441, 255, (2001).

[20] R. A. Bagnold, Proc. R. Soc. London Ser. A, 255, 49 , (1954).

[21] H. Henein, J. K. Brimacombe, and A. P. Watkinson, Metall. Trans. B, 14B, 191, (1983).

[22] M. Nakagawa, S. A. Altobelli, A. Caprihan, E. Fukushima, and E. K. Jeong, Exp. Fluids, 16, 54, (1993).

[23] J. Rajchenbach, E. Clément, and J. Duran, in Fractal Aspects of Materials, edited by F. Family, MRS Symposium Proceedings No. 367 (Materials Research Society, Pittsburgh, 1995), p. 525.

[24] C. M. Dury, G. H. Ristow, J. L. Moss, and M. Nakagawa, Phys. Rev. E, 57, 4491, (1998).

[25] K. Yamane, M. Nakagawa, S. A. Altobelli, T. Tanaka, and Y. Tsuji, Phys. Fluids, 10, 1419, (1998).
[26] J. Rajchenbach, in Physics of Dry Granular Media, edited by H. Hermann (Kluwer Academic, Dordrecht, 1998), p. 421.

[27] A. Daerr, and S. Douady, Nature, 399, 241 (1999).

[28] R. Khosropour, E. Valachovic, and B. Lincoln, Phys. Rev. E, 62, 807, (2000).

[29] P. -A. Lemieux, and D. J. Durian, Phys. Rev. Lett. 85, 4273 (2000).

[30] A. V. Orpe and D. V. Khakhar, Phys. Rev. E, 64, 031302 (2001).

[31] D. Bonamy, B. Faucherand, M. Planelle, F. Daviaud and L. Laurent, in Powders and Grains, edited by Y. Kishino (Swets and Zeitlinger, Lisse, 2001), 463.

[32] N. Jain, J. M. Ottino and R. M. Lueptow, Phys. Fluids, 14, 572 (2002).

[33] T. S. Komatsu, S. Inagaki, N. Nakagawa and S. Nasuno, Phys. Rev. Lett. 86, 1757 (2001). 